Wagner, Stefan; Lucka, Felix; Burger, Martin; Grasedyck, Lars; Haueisen, Jens;

Wolters, Carsten H.:

\title{
Comparison of direct and reciprocal forward modeling approaches in EEG source analysis
}

Zuerst erschienen in: Biomedical Engineering = Biomedizinische Technik. - Berlin [u.a.] : de Gruyter. - 57 (2012), Suppl. 1, Track-M, p. 310.

Erstveröffentlichung: $\quad$ 2012-09-04

ISSN (online): $\quad$ 1862-278X

ISSN (print): $\quad$ 0013-5585

DOI: $\quad 10.1515 / \mathrm{bmt}-2012-4069$

[Zuletzt gesehen: 2019-08-15]

„Im Rahmen der hochschulweiten Open-Access-Strategie für die Zweitveröffentlichung identifiziert durch die Universitätsbibliothek IImenau."

"Within the academic Open Access Strategy identified for deposition by Ilmenau University Library."

„Dieser Beitrag ist mit Zustimmung des Rechteinhabers aufgrund einer (DFGgeförderten) Allianz- bzw. Nationallizenz frei zugänglich."

"This publication is with permission of the rights owner freely accessible due to an Alliance licence and a national licence (funded by the DFG, German

DFG

Research Foundation) respectively." 


\title{
Comparison of direct and reciprocal forward modeling approaches in EEG source analysis
}

\author{
S. Wagner', F. Lucka ${ }^{1}$, M. Burger ${ }^{2}$, L. Grasedyck³ ${ }^{3}$ J. Haueisen ${ }^{4}$ and C.H. Wolters ${ }^{1}$ \\ 1 Institute for Biomagnetism and Biosignalanalysis, University of Muenster, 48149, Muenster, Germany \\ ${ }^{2}$ Institute for Computational and Applied Mathematics, University of Muenster, 48149 Muenster, Germany \\ ${ }^{3}$ Institute for Geometrical and Practical Mathematics (IGPM), RWTH Aachen, Templergraben 55, 52056 \\ Aachen, Germany \\ ${ }^{4}$ Institute of Biomedical Engineering and Informatics, IImenau University of Technology, 98693 IImenau, \\ Germany
}

\begin{abstract}
Our presentation will focus on a comparison between the reciprocal or adjoint approach (AA) (Vallaghe et al., 2009) and the partial integration (PI) direct approach using the transfer matrix approach (Wolters et al., 2004; Vorwerk, 2011) for realistic forward modeling in EEG source analysis.

Following (Vallaghe et al., 2009), the AA is deduced using the adjoint method and Riesz representation theorem is used in order to depict the connection between the AA and the EEG forward problem. Next, the PI is derived and used for a comparison with the AA (Wagner 2011). In order to investigate realistically shaped head models including tissue inhomogeneities and anisotropy, the finite element method (FEM) is used for numerical realization of both forward modeling approaches. The underlying theory will be discussed and the connection between lead field approach, Helmholtz' principle of reciprocity and the AA will be demonstrated in detail.

By means of a validation of our implementation in tetrahedral as well as hexahedral anisotropic multicompartment spherical shell models, an estimation of the computational complexity and a derivation of closed formulas, we will show that the AA and PI are essentially identical (Wagner, 2011).

However, the AA can be used to calculate and investigate lead field sensitivity distributions that serve as sensitivity maps for the considered pairs of electrodes (lead) in EEG source analysis.

Sensitivity maps can be used to (1) assess the influence of an arbitrary dipolar source on the potential difference between a fixed lead at a glance; (2) identify the orientation of certain dipoles needed to maximize the potential difference measured between the considered lead in an intuitive way; (3) visually predict the differences in forward modeling when extending a head model from a standard homogenized threecompartment model to a more realistic one.
\end{abstract}

\section{Literature}

[1] Vallaghe, S., Papadopoulo, T., Clerc, M., The adjoint method for general EEG and MEG sensorbased lead field equations, Phys.Med.Biol., 54, pp. 135-147, (2009).

[2] Vorwerk, J., Comparison of numerical approaches to the EEG forward problem, Diploma Thesis (Mathematics), Institute for Biomagnetism and Biosignalanalysis and Institute for Computational and Applied Mathematics, University of Muenster, (2011).

[3] Wagner, S., An adjoint FEM approach for the EEG forward problem, Diploma Thesis (Mathematics), Institute for Biomagnetism and Biosignalanalysis and Institute for Computational and Applied Mathematics, University of Muenster, (2011).

[4] Wolters, C.H., Grasedyck, L., Hackbusch, W., Efficient computation of lead field bases and influence matrix for the FEM-based EEG and MEG inverse problem, Inverse Problems, 20(4), pp. 1099-1116, (2004). 